\title{
Up-Regulation in Expression of Vesicular Glutamate Transporter 3 in Substantia Nigra but Not in Striatum of 6-Hydroxydopamine-Lesioned Rats
}

\author{
E.K.Y. Chung ${ }^{\text {a }}$ L.W. Chen ${ }^{\text {b Y.S. Chan }}{ }^{\text {C K.K.L. Yung }}{ }^{\text {a }}$ \\ a Department of Biology, Hong Kong Baptist University, Kowloon Tong, Hong Kong; ' ${ }^{\mathrm{b}}$ Institute of Neurosciences, \\ Fourth Military Medical University, Xian, and ' Department of Physiology and Research Center of Heart, Brain, \\ Hormone and Healthy Aging, University of Hong Kong, Hong Kong, PR China
}

\section{Key Words}

Animal model of Parkinson's disease $\cdot$ Neurotoxin •

Degeneration of dopaminergic neurons $\cdot$ Vesicular

glutamate transporters $\cdot$ Basal ganglia

\begin{abstract}
Overactivity of the glutamatergic system is suggested to be closely related to the onset and pathogenesis of Parkinson's disease. Vesicular glutamate transporters (VGLUT1, T2 and T3) are a group of glutamate transporters in neurons that are responsible for transporting glutamate into synaptic vesicles and they are key elements for homeostasis of glutamate neurotransmission. The present study was aimed to investigate the expression of VGLUT1, T2 and T3 proteins after the onset of Parkinson's disease. A rat model of Parkinson's disease, the 6-hydroxydopamine-lesioned rat, was employed. Immunocytochemistry revealed that VGLUT1, T2 and T3 immunoreactivity was not modulated in the striatum of the lesioned rat. Western blotting analyses also showed that there was no change in the expression of $\mathrm{T} 1, \mathrm{~T} 2$ and $\mathrm{T} 3$ proteins in the striatum. In contrast, no VGLUT1 protein was detected in the substantia nigra. After the lesion, levels of VGLUT2 immunoreactivity and protein were not modulated. Significant increase of VGLUT3 immunoreactivity was observed in the perikarya of GABAergic substantia nigra pars reticulata neurons (+14.7\%) although VGLUT3 protein was not modulated
\end{abstract}

in the nigral tissues. VGLUT3 in GABAergic neurons is suggested to play a role in GABA synthesis. The present results may therefore implicate that VGLUT1 and T2 are not modulated in the striatum and the substantia nigra of the 6-hydroxydopamine-lesioned rat and only VGLUT3 plays a role in pathogenesis of Parkinson's disease.

Copyright $\odot 2007$ S. Karger AG, Basel

\section{Introduction}

Parkinson's disease (PD) is a progressive neurological disorder and is characterized by a degeneration of dopaminergic neurons projecting from the substantia nigra pars compacta to the striatum of the basal ganglia. Previous studies $[6,7,13,17]$ have shown that changes in glutamate transmission may be involved in the pathogenesis of PD. One of the damaging effects after dopamine denervation is the alteration of the functional circuitry of the basal ganglia. The change is associated with an imbalance in the activity of the so called 'direct' and 'indirect' pathways $[2,3,13]$. The imbalance in fact reflects the changes in GABAergic and glutamatergic pathways of the basal ganglia. Thus the manifestation of PD symptoms has been shown to be related to the over-activity of the glutamatergic pathways $[6,7,13,16]$.

\section{KARGER}

Fax +4161306 1234

E-Mail karger@karger.ch

www.karger.com (c) 2007 S. Karger AG, Basel

$1424-862 X / 06 / 0155-0238 \$ 23.50 / 0$

Accessible online at:

www.karger.com/nsg
Ken K.L. Yung, DPhil

Department of Biology

Hong Kong Baptist University

Kowloon Tong, Hong Kong (PR China)

Tel. +852 34117 060, Fax +852 34115 995, E-Mail kklyung@hkbu.edu.hk 
By receiving excitatory cortical and thalamic inputs, the striatum is the input nuclei of the basal ganglia [38]. Release of glutamate in the striatum is modulated by nigrostriatal dopaminergic projections [10,30]. Because of the differential responses of the dopamine receptors on distinct populations of striatal projection neurons to the denervation, a complex alteration in the functional circuitry of the basal ganglia resulted in favor of the indirect pathway $[2,3,13]$. Activation of the indirect pathway, which involves the pathway from striatum to the output nuclei via the globus pallidus and the subthalamic nucleus, leads to increased firing of neurons in the output nuclei and causes inhibition in targets of the basal ganglia, thus constituting the underling cause of most of the symptoms that characterize $\operatorname{PD}[2,3,13]$.

It is recognized that transport of glutamate into synaptic vesicles in glutamatergic axonal terminals are regulated by a group of vesicular glutamate transporters, namely VGLUTs $[1,5,14,15,18,28,41-43]$. VGLUT-mediated glutamate uptake activity is dependent on the vesicular proton electrochemical gradient generated by the vesicular proton ATPase [4, 26, 31]. Previous studies have shown that the expression of the three vesicular glutamate transporters are highly differential in the basal ganglia, in which VGLUT1 and VGLUT2 are expressed in terminals immunostained by synaptophysin whereas VGLUT3 are expressed in glutamatergic, GABAergic, cholinergic and monoaminergic neurons in various regions in the brain $[18,24,29,36,37,39]$.

Increases in glutamate levels have been found in the striatum following dopamine denervation in an animal model of PD $[6,7,17]$. Overactivity in glutamatergic systems is then implicated in the pathogenesis of $\mathrm{PD}[6,7$, $16,17]$. The status of expression, i.e. alteration in expression of VGLUTs, if any, is one key element to understand the reasons of the onset of overactivity of the glutamatergic pathways in parkinsonian conditions, and also provides a new target for anti-parkinsonian therapies in the future. A previous study has found that there is no change in expression of VGLUT1 and VGLUT2 in the striatum after the onset of PD [34]. The objectives of the present project were to study the changes of expression of VGLUT1, VGLUT2 and VGLUT3 in an animal model of $\mathrm{PD}$, the 6-hydroxydopamine (6-OHDA)-lesioned rat. Changes in VGLUT proteins were investigated using immunofluorescence and Western blot analyses.

\section{Materials and Methods}

\section{Animals}

In the present study, 15 adult Sprague-Dawley rats (females, 150-200 g, University of Hong Kong) were used. Female rats were used in accordance to a previous study [48]. The handling of rats and all procedures involving the use of animals were approved in accordance with the Animals (Control of Experiments) Ordinance, Department of Health, Hong Kong and also approved by Committee on the Use of Human and Animal Subjects in Teaching and Research, Hong Kong Baptist University, and conformed to the Principles of Laboratory Animal Care [NIH publication No. 86-23, revised 1985]. All procedures performed by the investigators were aimed to minimize both animal number and suffering of the animals.

\section{6-Hydroxydopamine Lesion}

Rats were deeply anesthetized with sodium pentobarbital (60 $\mathrm{mg} / \mathrm{kg}$ i.p., sagittal). The rats then received unilateral stereotaxic injections of 6-hydroxydopamine (6-OHDA; $4 \mu \mathrm{l}, 3 \mathrm{mg} / \mathrm{ml}$ in $0.9 \%$ saline containing $0.2 \mathrm{mg} / \mathrm{ml}$ ascorbic acid) in the right medial forebrain bundle (coordinates bregma: -0.45 , midline: -0.09 , dura: -0.75 ) [32]. The injection was administrated within a period of $12 \mathrm{~min}$ and the needle of the Hamilton syringe was kept in situ for $10 \mathrm{~min}$ following deposit to prevent back filling along the injection tract. The animals were allowed to recover after surgery and kept in the animal house, Hong Kong Baptist University.

Rat Rotation Tests after 6-OHDA Lesion

One week after the 6-OHDA lesion, rats were tested for motor deficits in accordance to classical reports by Ungerstedt and coworkers [44, 45]. Apomorphine (1 mg/ml in saline, i.p., RBI) was injected to the rats. The numbers of rotations in a 30 -min test were noted for each rat. Those rats that turned with an average of over 7 turns per min were regarded as successfully lesioned animals.

\section{Tissue Preparation}

Rats were deeply anesthetized with sodium pentobarbital (60 $\mathrm{mg} / \mathrm{kg}$, i.p., sagittal). They were perfused transcardially with $50-$ $100 \mathrm{ml}$ of normal saline followed by $200 \mathrm{ml}$ fixative (3\% paraformaldehyde and $0.01 \%$ glutaraldehyde in $0.1 \mathrm{M}$ phosphate buffer (PB), $\mathrm{pH} 7.4)$. The brains were quickly removed from the skull after the perfusion and postfixed in $3 \%$ paraformaldehyde (in $0.1 \mathrm{M} \mathrm{PB}, \mathrm{pH} 7.4)$ for a few hours at $4^{\circ} \mathrm{C}$. Sections of the striatum and the substantia nigra $(70 \mu \mathrm{m})$ were cut on a vibrating microtome (Vibratome 1000, Technical Products International). The sections were collected in phosphate-buffered saline (PBS; $0.01 \mathrm{M}$, $\mathrm{pH} 7.4)$.

\section{Immunocytochemistry}

Immunocytochemistry was performed as previously described $[23,27]$. All the striatal and nigral sections for comparisons were incubated and reacted in a single reaction sequence to minimize variation in immunofluorescence. The striatal and nigral sections were incubated in primary antibody solutions against VGLUT1, VGLUT2 and VGLUT3 (all guinea pig origin, $1: 3,000$ in PBS supplemented with $2 \%$ normal goat serum and $0.1 \%$ Triton-X 100 (PBS-Triton); Chemicon International, Temcula, Calif., USA) for $24 \mathrm{~h}$ at room temperature. After incubation, 
the sections were washed $(3 \times$ PBS $)$ and incubated in Alexa 488 conjugated secondary antibody solutions (goat anti-guinea pig IgG, 1:200 in PBS-Triton; Molecular Probes) for $2 \mathrm{~h}$ at room temperature in dark. The sections were then washed $(3 \times \mathrm{PBS})$, mounted in mounting medium (Vectashield, Vector Labs) and examined on a confocal microscope (LSM 510, Zeiss).

In control experiments for immunofluorescence, where the primary or secondary antibodies were omitted from the reaction sequence, no VGLUT immunoreactivity was observed in those sections (data not shown).

Semiquantitative analyses of the fluorescence micrographs were performed in accordance to previous studies [22, 40]. Digital images of each group of animals, i.e., normal sham-operated striatum and substantia nigra from control rats, the nonlesioned striatum and substantia nigra from 6-OHDA-lesioned rats, and the lesioned striatum and substantia nigra from 6-OHDA-lesioned rats, were captured under the same parameters in the confocal microscope at high magnification (using a $63 \times$ lens). At least three sections of each animal were used for semiquantitative analyses. The fluorescent intensity of the confocal microscope images was determined by using an image analyzing software (Metamorph, Universal Imaging). The average grey values indicating the levels of VGLUT1 and VGLUT2 immunoreactivity in neuropilar elements and the level of VGLUT3 immunoreactivity in the cytoplasm were determined. Intensities were expressed as mean \pm SEM. Statistical comparisons between the groups were made by one-way analysis of variance (ANOVA, SPSS software).

In control experiments, the above reaction sequences were performed with the omission of either the primary or secondary antibody solutions or each of the primary antibodies in turn, in the reaction sequence.

\section{Western Blot Analysis}

The animals that were subjected to Western blot analysis were decapitated and the tissues of the striatum and substantia nigra were dissected as previously described [25]. The tissues were homogenized in a homogenizing buffer $(250 \mathrm{mM}$ sucrose, $50 \mathrm{M}$ imidazole hydrochloride, $2 \mathrm{~mm}$ sodium EDTA and $2 \mathrm{mM} \beta$-mercatoethanol, $\mathrm{pH} 7.2$ ) and centrifuged at $300 \mathrm{~g}$ for $10-15 \mathrm{~min}$. The supernatant was further centrifuged at $14,000 \mathrm{~g}$ for $15 \mathrm{~min}$. The final supernatant was stored at $-80^{\circ} \mathrm{C}$. The quantity of proteins in the supernatant was determined using a commercially available kit (Biorad protein assay kit). Proteins ( $60 \mu \mathrm{g} /$ well) were submitted to SDS-polyacrylamide gel electrophoresis and electroblotted onto nitrocellose membranes using a commercially available system (Bio-Rad Mini-protean 3). The membrane blots were subjected to Western blot analysis. Nonspecific-binding sites were blocked with $5 \%$ nonfat milk for $2 \mathrm{~h}$. The blots were then incubated overnight at $4{ }^{\circ} \mathrm{C}$ with one of the following primary antibodies in 2\% non-fat milk: VGLUT1 (1:2,000 in Tris-buffered saline (TBS) supplied with $2 \%$ non-fat skim milk, $0.05 \mathrm{M}, \mathrm{pH} 7.4$; Chemicon), VGLUT2 (1:2,000 in TBS; Chemicon) and VGLUT3 (1:1,000 in TBS; Chemicon), or $\beta$-actin (mouse monoclonal; 1:1,000 in TBS; Calbiochem). The membranes were then incubated in appropriate secondary antibodies (horseradish peroxidase-conjugated goat-anti-guinea pig IgG, 1:1,000 in TBS with 2\% non-fat skim milk or goat-anti-mouse IgG, 1:500 in TBS with 2\% non-fat skim milk, Zymed Laboratories) for $1 \mathrm{~h}$ at room temperature. Bands in the membranes were visualized using chemiluminescence detection (ECL Western blotting detection reagents;

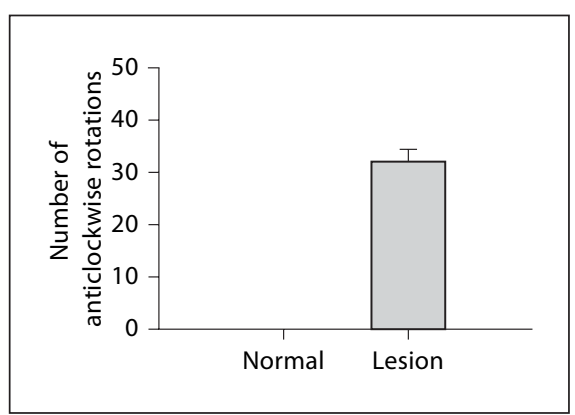

Fig. 1. Results of the apomorphine-induced rotation tests in rats before and after the 6-hydroxydopamine lesions are shown. No rotation was detected in rats before the lesion. Rotations were detected in rats after the lesion.

Amersham Pharmacia). Images of the bands were developed on films (Biomax X-ray film, Kodak) and captured by a computer with a scanner. Optical density of each band was then semiquantified by software (Metamorph) and statistically analyzed by oneway ANOVA. Distinct immunoreaction products for each VGLUT were revealed: VGLUT1 (approximately $60 \mathrm{kDa}$ ) [1], VGLUT2 (approximately $65 \mathrm{kDa}$ ) [28] and VGLUT3 (approximately 60 $\mathrm{kDa})[15]$.

Control for loading for Western blotting was performed in revealing $\beta$-actin proteins using either striatal or nigral tissues of the same animals. Specific bands of $45 \mathrm{kDa}$ were revealed in each of the trials.

\section{Results}

\section{Rat Rotation}

No contralateral rotation was detected in rats before being 6-OHDA-lesioned (fig. 1). After the lesion, rotations were detected (fig. 1).

\section{No Change in Expression of VGLUTs in the Striatum after Dopamine Denervation}

At low magnification, VGLUT1 immunoreactivity was seen in the neuropil of the striatum (fig. 2). The fiber bundles and neuronal cell bodies were immuno-negative (fig. 2). No difference was found in the expression pattern and intensity of VGLUT1 between the striatum from control animals, nor from the non-lesioned side and lesioned side of the 6-OHDA-lesioned animals (fig. 2c). At higher magnification, VGLUT1-immunoreactive puncta were clearly observed throughout the striatal sections. The neuronal perikarya were unstained (fig. $2 \mathrm{~d}-\mathrm{f}$ ). Semiquantitative analyses revealed that no significant difference in the intensity of VGLUT1 immunoreactivity of the 


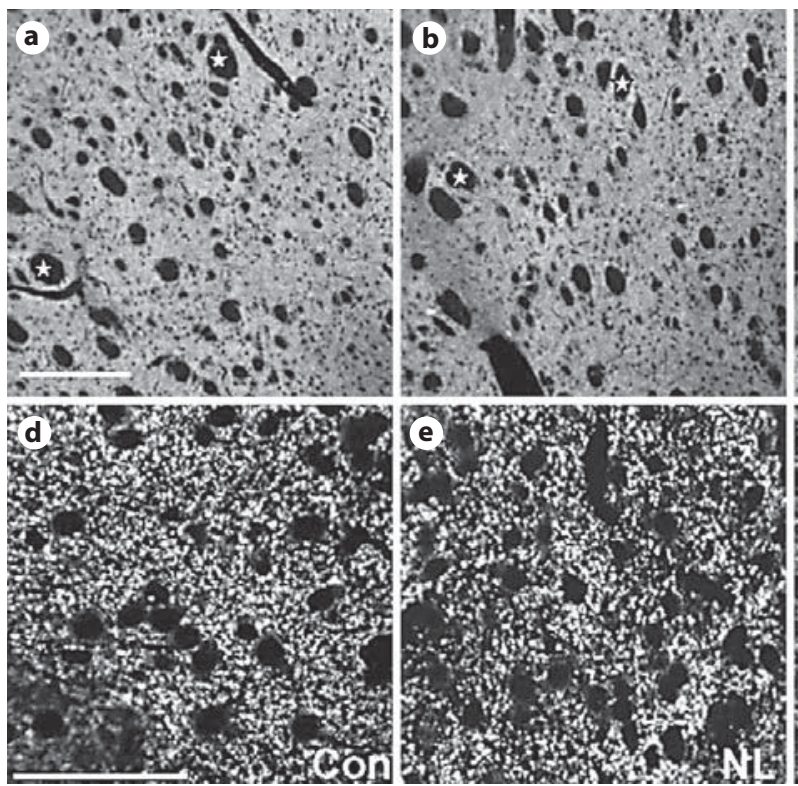

Fig. 2. Comparison of VGLUT1 immunoreactivity in the striatum of sham-operated control and unilateral 6-hydroxydopamine-lesioned rats. a-c Micrographs at low magnification show that VG immunoreactivity is predominantly found in the neuropil of the striatum. The fiber bundles are immuno-negative (some are indicated by white stars). The levels of VGLUT1 immunoreactivity in control striatum (a), non-lesioned striatum (b), and lesioned striatum (c) are similar. At higher magnification, neuronal cell bod-
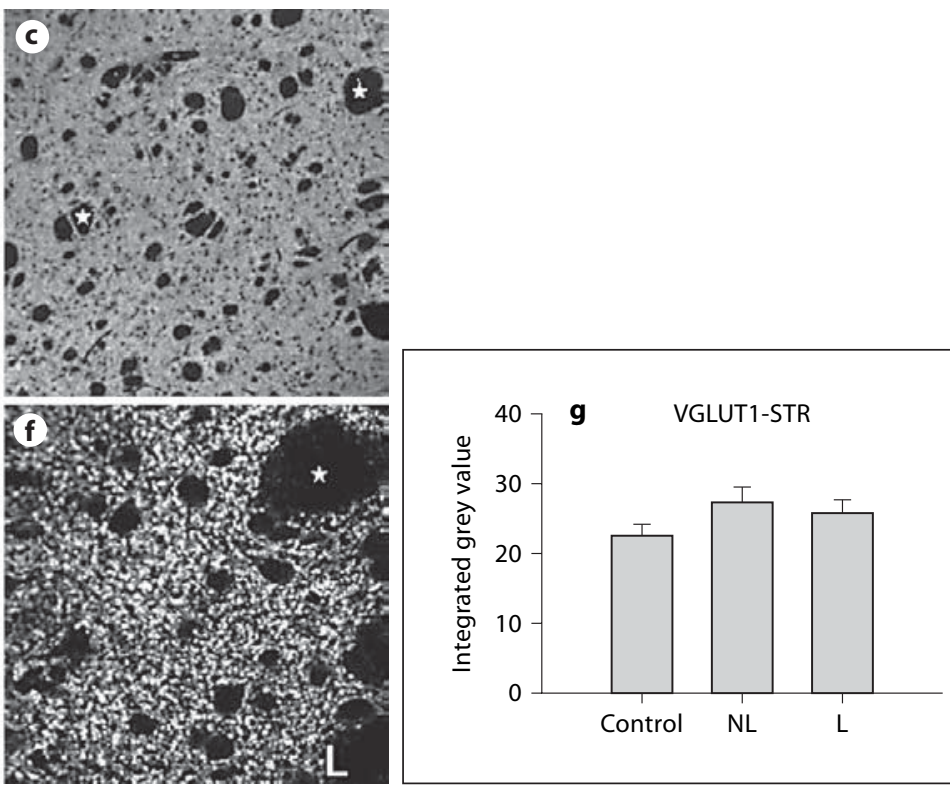

ies in the striatum are immuno-negative. Similarly, similar levels of VGLUT1 immunoreactivity are found in three groups of striatal sections. Semi-quantitative analysis shows that there is no significant difference in level of VGLUT1 immunoreactivity between the control and lesioned animals $(\mathbf{g})$. Mean \pm SEM is shown. Scale bars: $200 \mu \mathrm{m}$ (in a, for $\mathbf{a}-\mathbf{c}$ ); $50 \mu \mathrm{m}$ (in $\mathbf{d}$, for $\mathbf{d}-\mathbf{f}$ ). Con $=$ Control; $\mathrm{NL}=$ no lesion $; \mathrm{L}=$ lesion.
Table 1. Summarized results of the semiquantitative analysis of levels of immunofluorescence of the vesicular glutamate transporters in the regions of striatum and substantia nigra of the normal and lesioned animals

\begin{tabular}{|c|c|c|c|c|}
\hline & $\begin{array}{l}\text { Vesicular gluta- } \\
\text { mate transporters }\end{array}$ & Control & $\begin{array}{l}\text { Non-lesioned } \\
\text { side }(\mathrm{NL})\end{array}$ & $\begin{array}{l}\text { Lesioned } \\
\text { side }(\mathrm{L})\end{array}$ \\
\hline \multirow[t]{3}{*}{ Striatum } & VGLUT1 & $22.77 \pm 1.12$ & $21.34 \pm 0.27$ & $21.66 \pm 0.35$ \\
\hline & VGLUT2 & $19.46 \pm 1.55$ & $2.19 \pm 1.18$ & $18.80 \pm 0.65$ \\
\hline & VGLUT3 & $43.41 \pm 2.97$ & $44.34 \pm 1.70$ & $39.25 \pm 2.18$ \\
\hline \multirow[t]{2}{*}{ Substantia nigra } & VGLUT2 & $28.11 \pm 3.55$ & $28.41 \pm 1.54$ & $26.46 \pm 2.00$ \\
\hline & VGLUT3 & $28.87 \pm 0.75$ & $32.67 \pm 1.94$ & $\begin{array}{c}37.48 \pm 1.12^{*} \\
(\uparrow 14.72 \pm 5.94 \%)\end{array}$ \\
\hline
\end{tabular}

A total of 45 images from 15 animals were taken for each transporter in each region. The relative intensity of the images was expressed as the means \pm SEM. The values of the lesioned side were compared with both the non-lesioned side and the control. ${ }^{*} \mathrm{p}<$ 0.05 . control, non-lesioned and lesioned striatum (number of animals in each group [n] $=5$; fig $2 \mathrm{~g}$, table 1 ).

The distribution of VGLUT2 immunoreactivity was also uniformly throughout the striatum and was similar to the immunoreactivity for VGLUT1 (fig. 3). At low magnification, no observable difference in expression pattern and intensity for VGLUT2 immunoreactivity was noticed between the three groups of striatal sections (fig. 3a-c). At higher magnification, fine VGLUT2-positive puncta were observed throughout the striatum. The 

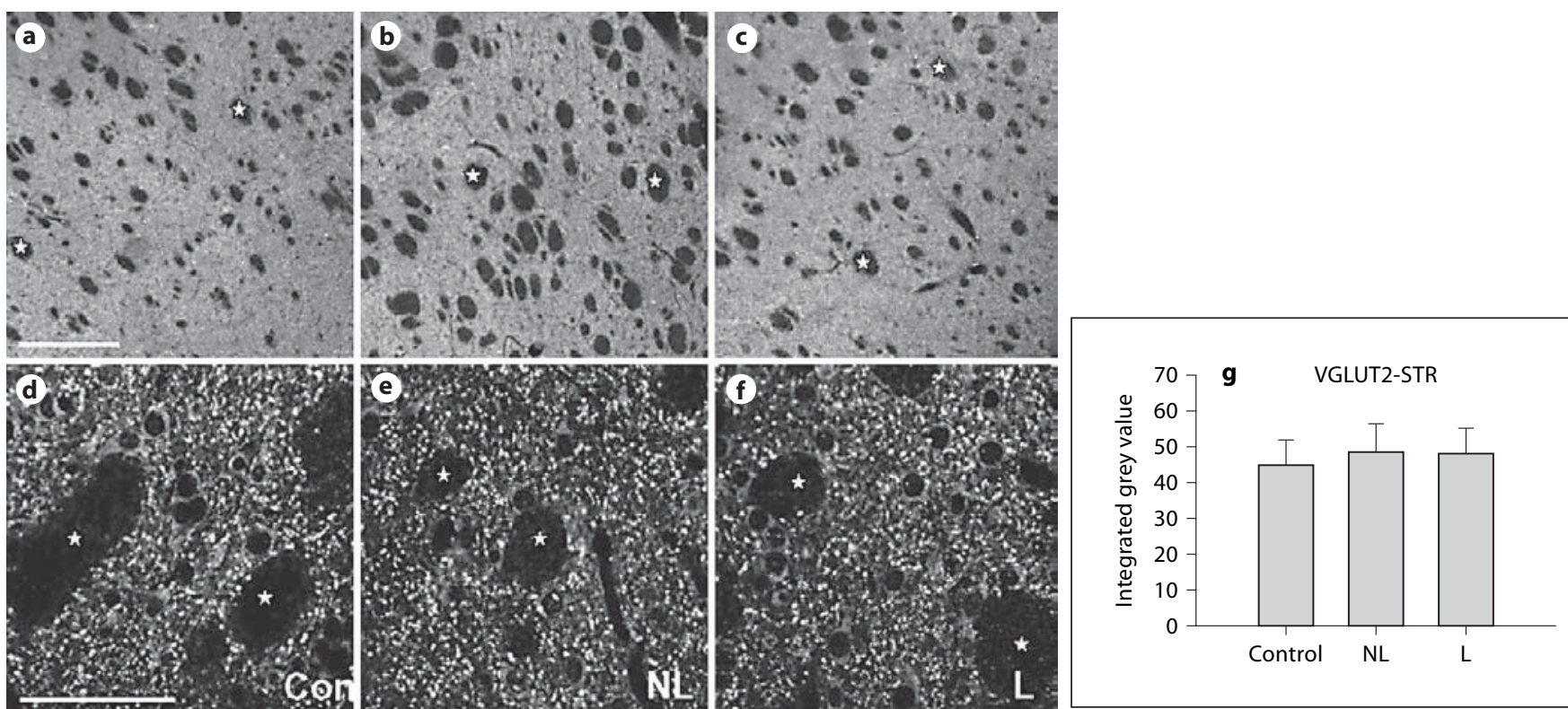

Fig. 3. Comparison of VGLUT2 immunoreactivity in the striatum of sham-operated control rat and unilateral 6-hydroxydopaminelesioned rat. Similarly, VGLUT2 immunoreactivity is predominantly found in the neuropil of the striatum $(\mathbf{a}-\mathbf{c})$. The fiber bundles are immuno-negative (some are indicated by white stars). The levels of VGLUT2 immunoreactivity in control striatum (a), non-lesioned striatum (b) and lesioned striatum (c) are similar. At higher magnification, neuronal cell bodies in the striatum are

immuno-negative. Similar levels of VGLUT2 immunoreactivity are found in control (d), non-lesioned (e) and lesioned striatum (f). Semiquantitative analysis shows that there is no significant difference in level of VGLUT2 immunoreactivity between the three groups $(\mathbf{g})$. Mean \pm SEM is shown. Scale bars: $200 \mu \mathrm{m}$ (in a, for $\mathbf{a}-\mathbf{c}$ ); $50 \mu \mathrm{m}$ (in d, for $\mathbf{d}-\mathbf{f}$ ). Con = Control; $\mathrm{NL}=$ no lesion; $\mathrm{L}=$ lesion.

neuronal perikarya in the striatum were immuno-negative for VGLUT2 (fig. 3d-f). Semiquantitative analyses also revealed that there was no significant difference of the intensity of VGLUT2 immunoreactivity in the lesioned side of striatum compared to the control and nonlesioned striatum ( $\mathrm{n}=4$; fig. $3 \mathrm{~g}$; table 1$)$.

At low magnification, VGLUT3 immunoreactivity was seen to be abundant in the striatum. There were no significant differences in the patterns of distribution and immunofluorescence intensity of VGLUT3 immunoreactivity between the normal, non-lesioned and lesioned striatum (fig. 4a-c). At higher magnification, VGLUT3 immunoreactivity was primarily found in cytoplasm of neurons (fig. $4 \mathrm{~d}-\mathrm{f}$ ) that were presumed to be striatal interneurons in accordance to their morphological characters [8]. No significance difference in the intensity of immunoreactivity in the cytoplasm of VGLUT3-immunoreactive perikarya was seen between the three groups ( $\mathrm{n}=5$; fig. 4g; table 1).

Results of the Western blot analyses are shown in figure 5. After 6-OHDA lesion, no significant differences in the expression of VGLUT1 (fig. 5a, b), VGLUT2 (fig. 5c,

d) and VGLUT3 (fig. 5e, f) proteins were found, respectively, between the control and the lesioned and nonlesioned striatum.

\section{VGLUT3 Proteins Were Up-Regulated in the}

Substantia Nigra after Dopamine Denervation

In the substantia nigra, no VGLUT1 immunoreactivity was detected (data not shown). In contrast, VGLUT2 immunoreactivity was found to be abundant in the neuropil of the substantia nigra pars compacta and pars reticulata (fig. 6a-c). Among the three groups of nigral sections, no observable differences in the distribution and intensity of VGLUT2 immunoreactivity were seen (fig. 6a-c). At higher magnification, VGLUT2-immunoreactive puncta were abundant in the pars reticulata where they were found primarily around unstained neuronal perikarya (fig. $6 \mathrm{~d}-\mathrm{f}$ ). Semi-quantitative analyses revealed that there was no significant difference in the levels of VGLUT2 immunoreactivity between the three groups of nigral sections ( $\mathrm{n}=3$; fig. $6 \mathrm{~g}$; table 1$)$.

In contrast, immunoreactivity for VGLUT3 was found in neuronal perikarya that were localized in the substan- 

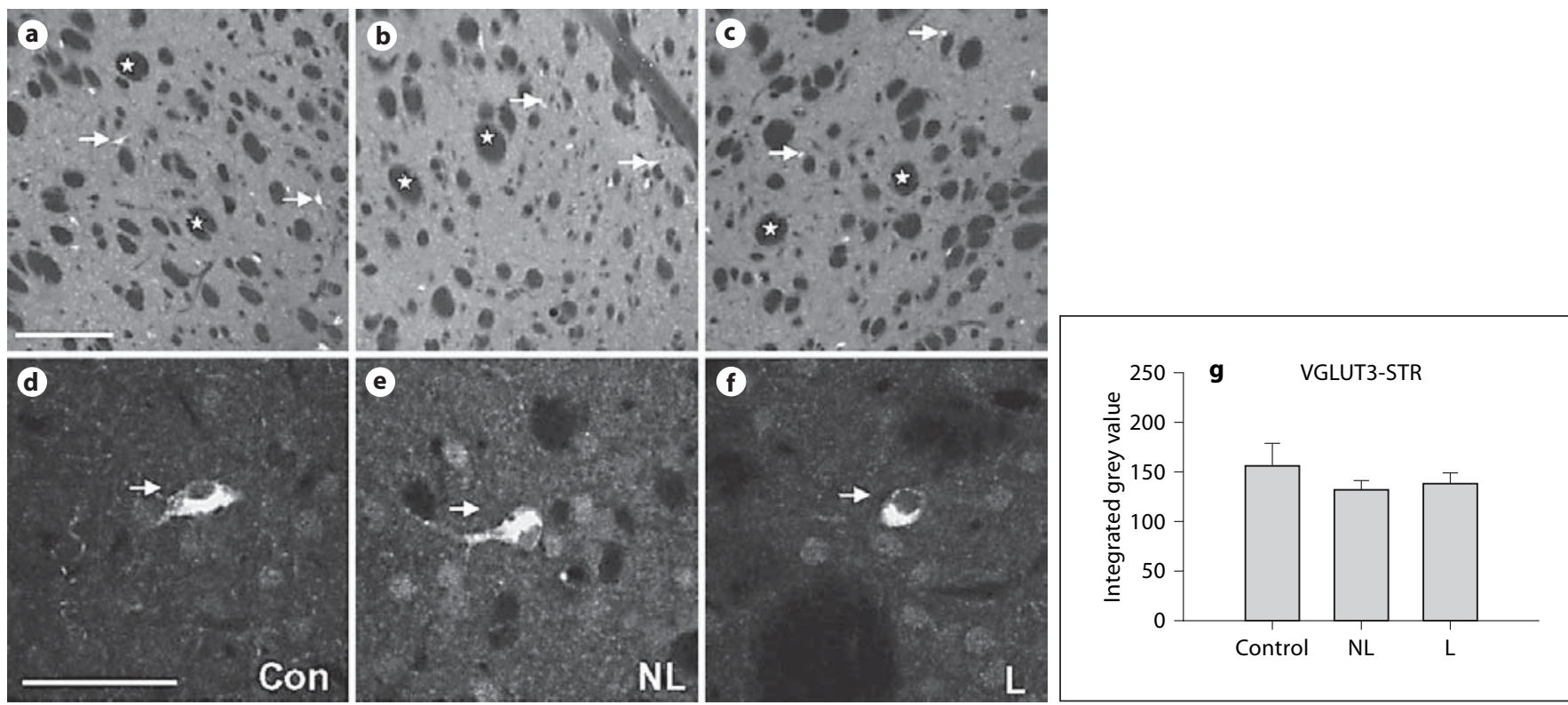

Fig. 4. Comparison of VGLUT3 immunoreactivity in the striatum of sham-operated control rat and unilateral 6-hydroxydopaminelesioned rat. At low magnification, VGLUT3 immunoreactivity is primarily found in perikarya in the striatum (a-c; some are indicated by white arrows). The fiber bundles are immuno-negative. Similar levels of VGLUT3 immunoreactivity have been found in control and in two sides of striatum of the lesioned animals $(\mathbf{a}-\mathbf{c})$. At higher magnification, perikarya of neurons in the striatum display intense VGLUT3 immunoreactivity (white arrows). In perikarya of striatal neurons, similar levels of VGLUT3 immunoreactivity are found among the control and the two sides of striatum (d-f). Semiquantitative analysis shows that there is no significant difference between the levels of VGLUT3 immunoreactivity (g). Mean \pm SEM is shown. Scale bars: $200 \mu \mathrm{m}$ (in a, for $\mathbf{a}-\mathbf{c}$ ); $50 \mu \mathrm{m}$ (in d, for $\mathbf{d}-\mathbf{f}$ ). Con = Control; NL = no lesion; $\mathrm{L}=$ lesion. tia nigra pars compacta and the pars reticulata (fig. 7a-c). Since the dopaminergic neurons in SNc were selectively depleted in the lesioned animals, VGLUT3-positive perikarya were only observed in the pars reticulata of the lesioned side (fig. 7c). More intensely labeled VGLUT3 immunoreactivity was seen in the lesioned substantia nigra when compared to those of the normal animal and the nonlesioned nigra (fig. $7 \mathrm{a}-\mathrm{c}$ ). At higher magnification, a significant higher level of VGLUT3 immunoreactivity was observed in the cytoplasm of neurons in the lesioned substantia nigra (fig. 7d-f). Semiquantitative analyses revealed that there was a significant increase in the level of VGLUT3 immunoreactivity in the reticulata neurons of the lesioned nigra $(+14.72 \pm 5.94 \% ; \mathrm{n}=5 ; \mathrm{p}<0.05$; fig. $7 \mathrm{~g}$; table 1).

In contrast, unlike the above results of immunoreactivity, Western blotting analyses showed that there was no significant difference in the expression of VGLUT2 and VGLUT3 proteins between the three groups of nigral tissues $(\mathrm{n}=3$; fig. $8 \mathrm{a}-\mathrm{d})$.

Up-Regulation of VGLUT3 in Substantia Nigra

\section{Discussion}

Dopamine Denervation Does Not Alter the Expression of VGLUTs in the Striatum

The patterns of distribution of VGLUT1 and VGLUT2 immunoreactivity in the striatum is consistent with a previous study [34]. There are regional distributions of VGLUT1 and VGLUT2 in the brain. Previous studies show that VGLUT1 mRNA expression is primarily associated with cortical afferents, whereas VGLUT2 mRNA expression is associated with intralaminar thalamic afferents $[19,28,46]$. The present results also show that VGLUT2 immunoreactivity is predominantly found in the substantia nigra whereas VGLUT1 immunoreactivity is not detected in the nigra.

The striatum is the main target of nigral dopaminergic input and also receives two major glutamatergic projections originating from the cerebral cortex and thalamus $[9,38]$. Striatal neurons including medium spiny neurons in the striatum receive convergent synaptic inputs from nigral dopaminergic projections and cortical 
Fig. 5. Western blotting analyses of the protein levels of VGLUT1, T2 and T3 in the striatum. a, b VGLUT1 proteins are revealed (approximately $60 \mathrm{kDa}$ ). Same levels of VGLUT1 proteins are found and no significant difference is found in the control, non-lesioned (NL) and lesioned (L) striatal tissues. c, d Similarly, VGLUT2 proteins are revealed (approximately 65 $\mathrm{kDa}$ ). No significant change in VGLUT2 proteins is found in the control, nonlesioned and lesioned striatal tissues. e, f VGLUT3 proteins are revealed (approximately $60 \mathrm{kDa}$ ). No significant difference is observed between the control, non-lesioned and lesioned striatal tissues. g $\beta$-Actin proteins were also revealed (approximately $45 \mathrm{kDa}$ ) as the control for protein loading.

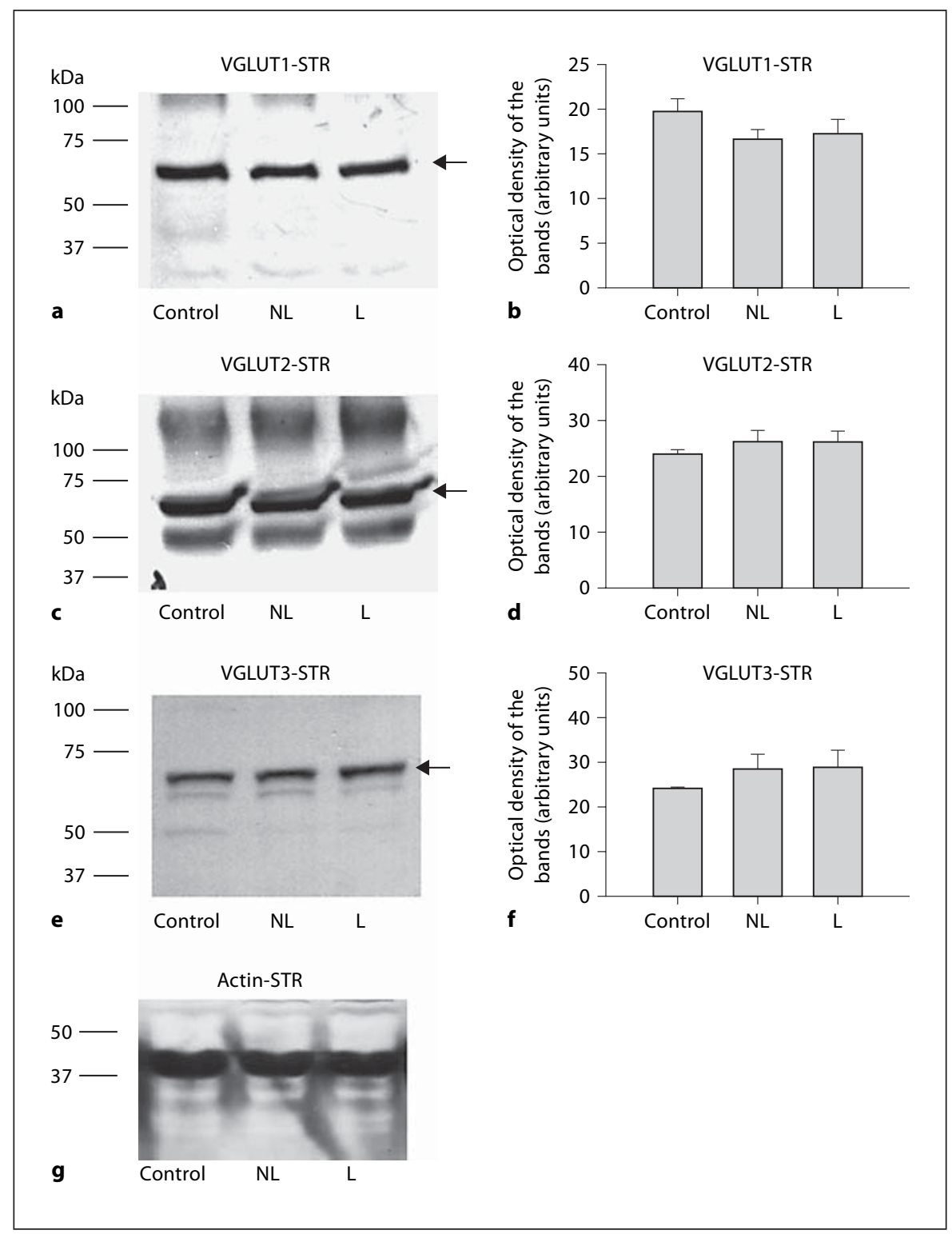

glutamatergic projections $[9,38]$. Dopamine denervation in PD induces an increase in corticostriatal glutamatergic transmission $[6,7,10,16,17,30]$ and an increase in the extracellular glutamate levels in the striatum [34].

Expression of VGLUT1 and VGLUT2 can regulate the quantal size of glutamate synaptic vesicles $[12,33,47]$. Overexpression of VGLUT1 in cultured glutamatergic neurons increases the quantal size of glutamate synaptic vesicles [47]. Therefore, it is believed that the expression of VGLUT1 and T2 may play an important role in controlling the synaptic transmission and also play crucial roles in regulation of the level of extrasynaptic glutamate.

The present study shows that lesions of nigral dopamine neurons do not significantly affect the expression of either VGLUT1 or VGLUT2 in the striatum. Therefore, it is suggested that the over-spills of glutamate after dopamine denervation may not relate to any modification in the expression of the VGLUTs, thus not relate to the packing of the glutamate in synaptic vesicles. The present results however, do not preclude the possibility that there is change in activity of either the corticostriatal and thalamostriatal pathways. 

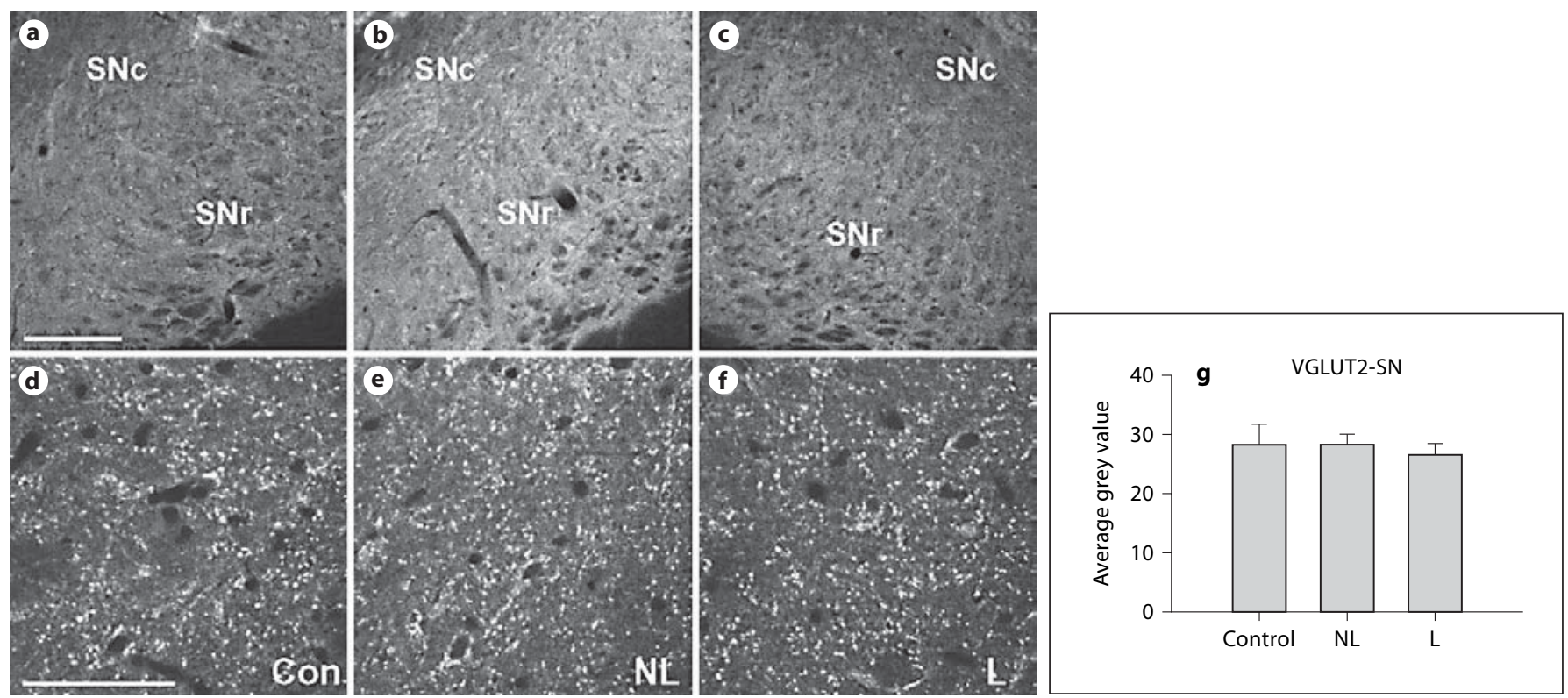

Fig. 6. Comparison of VGLUT2 immunoreactivity in the substantia nigra of sham-operated control rat and unilateral 6-hydroxydopamine-lesioned rat. VGLUT2 immunoreactivity is predominantly found in the neuropil of the nigra. The levels of VGLUT2 immunoreactivity are similar among all nigral sections. At higher magnification, VGLUT2 immunoreactivity is only observed in the neuropil. Similar levels of VGLUT2 immunoreactivity are found in the nigral sections (d-f). Semiquantitative analysis shows that there is no significant difference in level of VGLUT2 immunoreactivity between the control, non-lesioned and lesioned nigral sections $(\mathbf{g})$. Mean \pm SEM is shown. Scale bars: $200 \mu \mathrm{m}$ (in $\mathbf{a}$, for $\mathbf{a}-\mathbf{c}$ ); $50 \mu \mathrm{m}$ (in $\mathbf{d}$, for $\mathbf{d}-\mathbf{f}$ ). Con = Control; $\mathrm{NL}=$ no lesion; $\mathrm{L}=$ lesion; $\mathrm{SNc}=$ substantia nigra pars compacta; $\mathrm{SNr}=$ substantia nigra pars reticulata.
There is evidence to show that a large proportion of the increased extrasynaptic glutamate in the striatum after dopamine denervation is not arising from nonvesicular release [34]. The increase is depended on the balance between the release processes and glutamate transporter activities [34]. Previous studies suggest that synaptic release of glutamate from axonal terminals by exocytosis of synaptic vesicles is not the only mechanism supplying the extracellular fluid with glutamate, i.e. most of the released glutamate is of non-vesicular origin $[11,20]$. One possible source for the nonvesicular released glutamate is through the cysteine-glutamate exchangers $[11,20]$.

The present results indicate that VGLUT3 immunoreactivity is primarily expressed by striatal interneurons, the presumed striatal cholinergic neurons $[15,42]$. Dopamine denervation also does not modulate the protein expression of VGLUT3 nor the immunoreactivity for VGLUT3 in the region of the striatum or in perikarya of striatal cholinergic neurons. In the striatum, it is suggested that the release of glutamate is modulated by presynaptic acetylcholine receptors and striatal cholinergic interneurons play crucial roles in modulation of the functions of dopamine on medium spiny neurons
[21]. Acetylcholine, from striatal cholinergic neurons, exhibits opposing effects to dopamine on the striatonigral and striatopallidal neurons [21]. The striatal cholinergic interneurons expressing VGLUT3 raised the possibility that glutamate may be simultaneously co-released with acetylcholine $[15,42]$. However, the physiological roles of VGLUT3 in cholinergic neurons and their roles in onset of PD symptoms remain to be established.

\section{VGLUT3 but Not VGLUT2 Expression Is Up-Regulated in the Substantia Nigra after 6-OHDA Lesion}

The present results also show that VGLUT2 but not T1 immunoreactivity is primarily found in the substantia nigra. It is believed that VGLUT2-expressing glutamatergic synapses may display a higher probability of glutamate release when compared to those of VGLUT1-expressing glutamatergic synapses $[14,18,46]$. Glutamatergic projections from the subthalamic nucleus to the substantia nigra are found to operate exclusively with VGLUT2 [46]. A previous study suggests that the high activity of VGLUT2-expressing glutamatergic projec- 

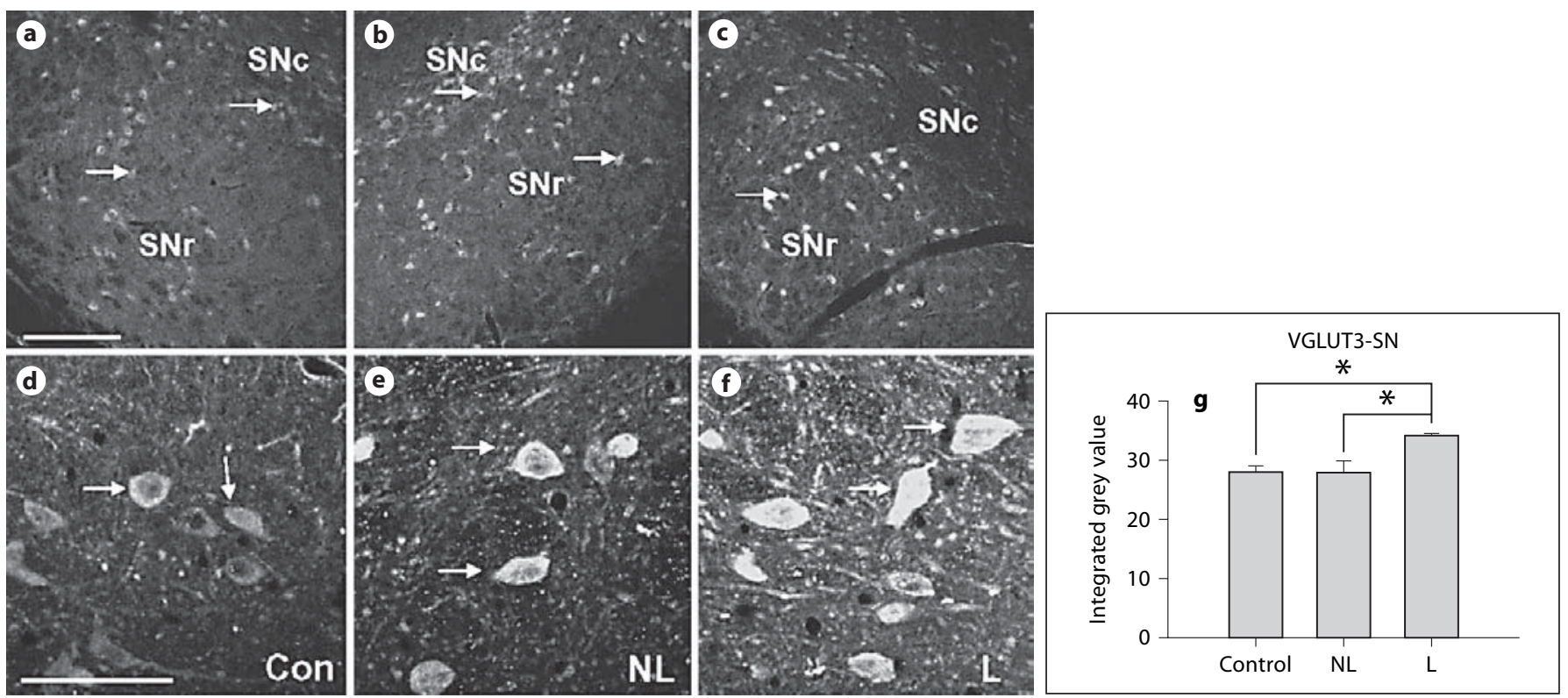

Fig. 7. Comparison of VGLUT3 immunoreactivity in the substantia nigra of sham-operated control rat and unilateral 6-hydroxydopamine-lesioned rat. At low magnification, VGLUT3 immunoreactivity is primarily found in perikarya in the substantia nigra pars compacta and pars reticulata in control and non-lesioned nigra (a, b, some are indicated by white arrows). In lesioned nigra, higher levels of VGLUT3 immunoreactivity are found in perikarya of the pars reticulata (c, white arrow). At higher magnification, perikarya of neurons in the substantia nigra pars reticulata display intense VGLUT3 immunoreactivity (d-f, some are indi- cated by white arrows). Similar levels of VGLUT3 immunoreactivity are found in the control and non-lesioned side (d-f), whereas a higher level of VGLUT3 immunoreactivity is found in the lesioned side (f). Semiquantitative analysis shows that a significantly higher level of VGLUT3 immunoreactivity is found in the lesioned side (g). Mean \pm SEM is shown. ${ }^{*} \mathrm{p}<0.05$. Scale bars: $200 \mu \mathrm{m}$ (in $\mathbf{a}$, for $\mathbf{a}-\mathbf{c}$ ); $50 \mu \mathrm{m}$ (in $\mathbf{d}$, for $\mathbf{d}-\mathbf{f}$ ). Con = Control; NL = no lesion; $\mathrm{L}=$ lesion; $\mathrm{SNc}=$ substantia nigra pars compacta; $\mathrm{SNr}=$ substantia nigra pars reticulata. tions of the subthalamic nucleus to the substantia nigra pars compacta may be implicated as a mechanism driving glutamate excitotoxicity of the dopaminergic neurons [35]. In PD, the loss of dopaminergic neurons in the compacta leads to an overactivity of the subthalamic nucleus which, in turn, causes further excitotoxic damage to vulnerable dopaminergic neurons. As a consequence, an increasing cycle of neuronal loss in the compacta may result in the development of PD [35]. However, the present results have demonstrated that there is no significant change in VGLUT2 expression in the substantia nigra. A homeostatic mechanism may exist to attenuate the excitatory effects of excess glutamate release by controlling the balance between the quantal content and the number of synaptic vesicles [12]. Another previous study demonstrates that an increase expression of VGLUTs in motoneurons leads to an increase in quantal content together with a compensatory decrease in the number of synaptic vesicles released, and these two processes result in a normal level of glutamate [12]. Although no change in the level of VGLUT2 expression was found in the present study, it does not preclude the possibility that there is an increase in glutamate vesicles released in the synapse.

Interestingly, the present study first demonstrates that there are up-regulations of VGLUT3 immunoreactivity in the reticulata neurons after the onset of PD. In the nigral tissues, no change of VGLUT3 protein is found by Western blotting. This discrepancy is likely due to the change in VGLUT3 is only found in the perikarya of reticulata neurons then the change is not sensitive enough detecting at the whole region level. The modulation of VGLUT3 expression has been found to be parallel to the change of activities of these neurons, i.e. the reticulata neurons are over-active in PD and the expression of VGLUT3 immunoreactivity is found to be upregulated. One possible explanation is that VGLUT3 is known to have a role in regulating intracellular storage pool of glutamate; the intracellular glutamate can be used for the synthesis of GABA [41]. Thus modulations of VGLUT3 expression may associate with changes in GABA synthe- 
Fig. 8. Western blotting analyses of the protein levels of VGLUT2 and T3 in the substantia nigra. a, b VGLUT2 proteins are revealed (approximately $65 \mathrm{kDa}$ ). No significant difference is found between the control, non-lesioned (NL) and lesioned (L) nigral tissues. c, d VGLUT3 proteins are revealed (approximately $60 \mathrm{kDa}$ ). No significant difference is found between the control, non-lesioned and lesioned nigral tissues. e $\beta$-Actin proteins were also revealed (approximately $45 \mathrm{kDa}$ ) as the control for protein loading.

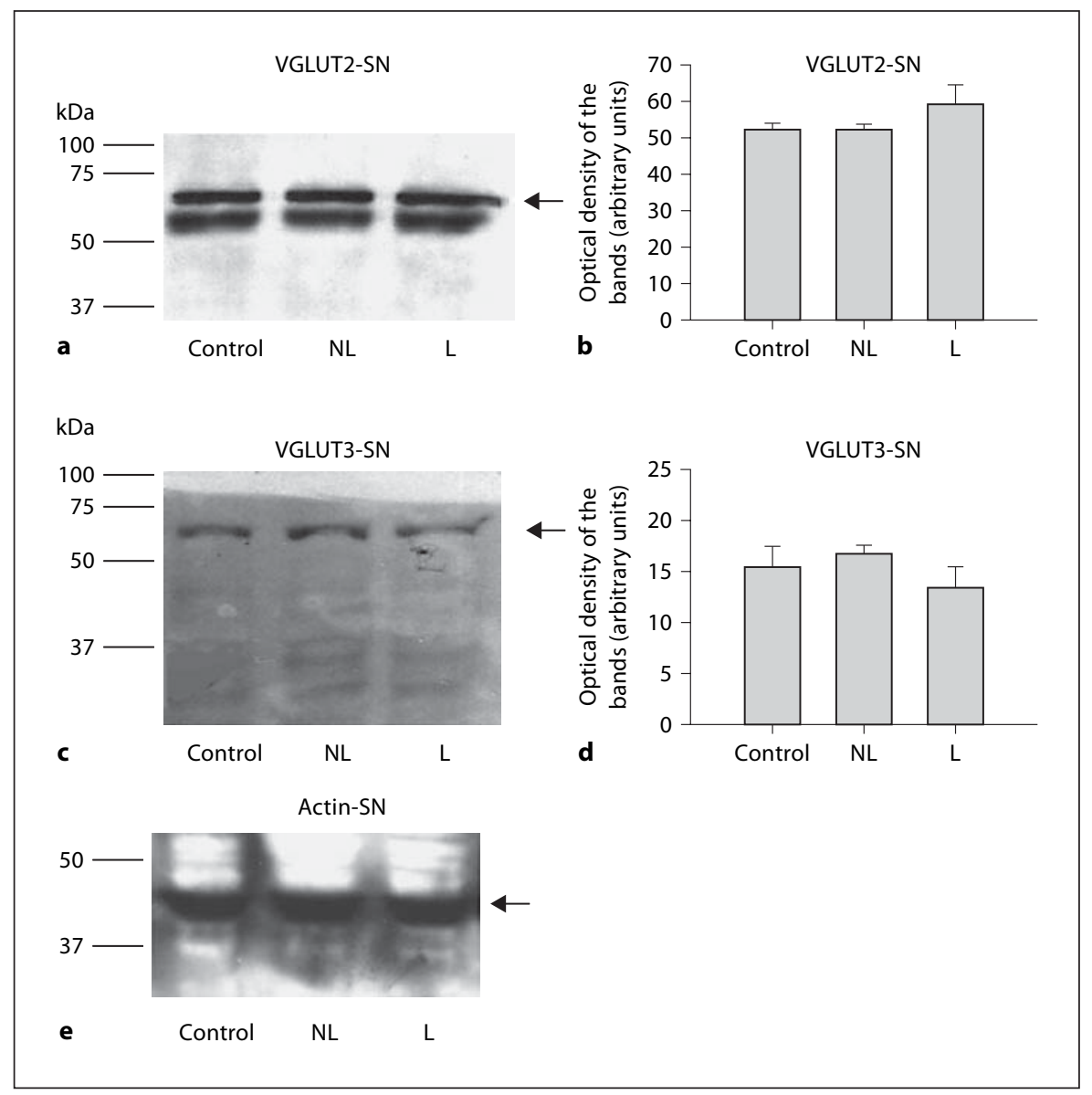

sis and release in the GABAergic reticulata neurons. The increase in GABA synthesis may in turn relate to he increases in activity of the reticulata neurons. The reticulata neurons are the output neurons of the basal ganglia that their over-activity result in hypokinetic symptoms of PD $[2,3,13]$. Therefore, the increase of VGLUT3 expression in the reticulate may be one key element that leads to the changes of GABAergic pathway in the basal ganglia in PD. VGLUT3 is therefore a target for pharmacological intervention of symptoms of PD.

\section{Acknowledgements}

The present work was supported by HKBU 2150/03M Research Grants Council, Hong Kong. Elaine Chung was supported by a postgraduate studentship. The authors would also like to thank Miss L.Y. Man and Miss Louise Ng for technical assistance.

\section{References}

Up-Regulation of VGLUT3 in Substantia Nigra
1 Aihara $\mathrm{Y}$, Mashima H, Onda H, Hisano S, Kasuya $\mathrm{H}$, Hori T, Yamada S, Tomura H, Yamada Y, Inoue I, Kojima I, Takeda J: Molecular cloning of a novel brain-type $\mathrm{Na}(+)$-dependent inorganic phosphate cotransporter. J Neurochem 2000;74:2622-2625.

2 Albin RL, Young AB, Penney JB: The functional anatomy of basal ganglia disorders. Trends Neurosci 1989;12:366-375.
3 Albin RL, Young AB, Penney JB: The functional anatomy of disorders of the basal ganglia. Trends Neurosci 1995;18:63-64.

4 Bai L, Xu H, Collins JF, Ghishan FK: Molecular and functional analysis of a novel neuronal vesicular glutamate transporter. J Biol Chem 2001;276:36764-36769.

5 Bellocchio EE, Reimer RJ, Fremeau RT Jr, Edwards RH: Uptake of glutamate into synaptic vesicles by an inorganic phosphate transporter. Science 2000;289:957-960. 
6 Blandini F, Greenamyre JT, Nappi G: The role of glutamate in the pathophysiology of Parkinson's disease. Funct Neurol 1996;11:3-15.

7 Blandini F, Porter RH, Greenamyre JT: Glutamate and Parkinson's disease. Mol Neurobiol 1996;12:73-94.

8 Bolam JP, Bennett BD: Microcircuitry of the neostriatum; in Ariano MA, Surmeier PJ (eds): Molecular and Cellular Mechanisms of Neostriatal Function. Austin, Landes Company, 1995, pp 1-19.

9 Bolam JP, Hanley JJ, Booth PA, Bevan MD: Synaptic organisation of the basal ganglia. J Anat 2000;196:527-542.

10 Calabresi P, Mercuri NB, Sancesario G, Bernardi G: Electrophysiology of dopamine-denervated striatal neurons. Implications for Parkinson's disease. Brain 1993;116:433452.

11 Danbolt NC: Glutamate uptake. Prog Neurobiol 2001;65:1-105.

12 Daniels RW, Collins CA, Gelfand MV, Dant J, Brooks ES, Krantz DE, DiAntonio A: Increased expression of the Drosophila vesicular glutamate transporter leads to excess glutamate release and a compensatory decrease in quantal content. J Neurosci 2004;24: 10466-10474.

13 DeLong MR: Primate models of movement disorders of basal ganglia origin. Trends Neurosci 1990;13:281-285.

14 Fremeau RT Jr, Troyer MD, Pahner I, Nygaard GO, Tran CH, Reimer RJ, Bellocchio EE, Fortin D, Storm-Mathisen J, Edwards RH: The expression of vesicular glutamate transporters defines two classes of excitatory synapse. Neuron 2001;31:247-260.

15 Gras C, Herzog E, Bellenchi GC, Bernard V, Ravassard P, Pohl M, Gasnier B, Giros B, El Mestikawy S: A third vesicular glutamate transporter expressed by cholinergic and serotoninergic neurons. J Neurosci 2002;22: 5442-5451.

16 Greenamyre JT: Glutamate-dopamine interactions in the basal ganglia: relationship to Parkinson's disease. J Neural Transm Gen Sect 1993;91:255-269.

17 Greenamyre JT: Glutamatergic influences on the basal ganglia. Clin Neuropharmacol 2001; 24:65-70.

18 Herzog E, Bellenchi GC, Gras C, Bernard V, Ravassard P, Bedet C, Gasnier B, Giros B, El Mestikawy S: The existence of a second vesicular glutamate transporter specifies subpopulations of glutamatergic neurons. J Neurosci 2001;21:RC181.

19 Hisano S, Nogami H: Transporters in the neurohypophysial neuroendocrine system, with special reference to vesicular glutamate transporters (BNPI and DNPI): a review. Microsc Res Tech 2002;56:122-131.

20 Jabaudon D, Shimamoto K, Yasuda-Kamatani Y, Scanziani M, Gahwiler BH, Gerber U: Inhibition of uptake unmasks rapid extracellular turnover of glutamate of nonvesicular origin. Proc Natl Acad Sci USA 1999;96: 8733-8738.
21 Kaneko S, Hikida T, Watanabe D, Ichinose $\mathrm{H}$, Nagatsu T, Kreitman RJ, Pastan I, Nakanishi S: Synaptic integration mediated by striatal cholinergic interneurons in basal ganglia function. Science 2000;289:633-637.

22 Lau WK, Lui PW, Wong CKC, Chan YS, Yung KKL: Differential expression of N-methyl-Daspartate receptor subunit messenger ribonucleic acids and immunoreactivity in the rat neostriatum during postnatal development. Neurochem Int 2003;43:47-65.

23 Lau WK, Yung WH, Chan YS, Yung KKL: Developmental assembly of GABA receptors in the rat substantia nigra; in Magill PJ, Bolam JP (eds): Basal Ganglia VIII. New York, Springer, 2005, pp 229-240.

24 Lin LH, Talman WT: Nitroxidergic neurons in rat nucleus tractus solitarii express vesicular glutamate transporter 3. J Chem Neuroanat 2005;29:179-191.

25 Lui PW, Yeung CW, Yung WH, Shi Y, Chen LW, Chan YS, Yung KKL: Ablation of gene expression of $\mathrm{N}$-methyl-D-aspartate receptor one by antisense oligonucleotides in striatal neurons in culture. Neurosignals 2005;14: 303-316.

26 Naito S, Ueda T: Characterization of glutamate uptake into synaptic vesicles. J Neurochem 1985;44:99-109.

27 Ng TKY, Yung KKL: Differential expression of $\mathrm{GABA}_{\mathrm{B}} \mathrm{R} 1$ and $\mathrm{GABA}_{\mathrm{B}} \mathrm{R} 2$ receptor immunoreactivity in neurochemically identified neurons of the rat neostriatum. J Comp Neurol 2001;433:458-470.

28 Ni B, Wu X, Yan GM, Wang J, Paul SM: Regional expression and cellular localization of the $\mathrm{Na}(+)$-dependent inorganic phosphate cotransporter of rat brain. J Neurosci 1995; 15 : 5789-5799.

29 Nickerson PA, Guerci A, El Mestikawy S, Semba K: Vesicular glutamate transporter 3 immunoreactivity is present in cholinergic basal forebrain neurons projecting to the basolateral amygdala in rat. J Comp Neurol 2006;498:690-711.

30 Onn SP, West AR, Grace AA: Dopamine-mediated regulation of striatal neuronal and network interactions. Trends Neurosci 2000;23: S48-S56.

31 Ozkan ED, Ueda T: Glutamate transport and storage in synaptic vesicles. Jpn J Pharmacol 1998;77:1-10.

32 Paxinos P, Watson C: The Rat Brain in Stereotaxic Coordinates, ed 2. New York, Academic Press, 1986.

33 Pothos EN, Larsen KE, Krantz DE, Liu Y, HaycockJW, Setlik W, Gershon MD, Edwards $\mathrm{RH}$, Sulzer D: Synaptic vesicle transporter expression regulates vesicle phenotype and quantal size. J Neurosci 2000;20:7297-7306.

34 Robelet S, Melon C, Guillet B, Salin P, Kerkerian-Le Goff L: Chronic L-DOPA treatment increases extracellular glutamate levels and GLT1 expression in the basal ganglia in a rat model of Parkinson's disease. Eur J Neurosci 2004:20:1255-1266.
35 Rodriguez MC, Obeso JA, Olanow CW: Subthalamic nucleus-mediated excitotoxicity in Parkinson's disease: a target for neuroprotection. Ann Neurol 1998;44:S175-S188.

36 Schafer MK, Varoqui H, Defamie N, Weihe E, Erickson JD: Molecular cloning and functional identification of mouse vesicular glutamate transporter 3 and its expression in subsets of novel excitatory neurons. J Biol Chem 2002;277:50734-50748.

37 Seal RP, Edwards RH: The diverse roles of vesicular glutamate transporter 3. Handb Exp Pharmacol 2006;137-150.

38 Smith Y, Bevan MD, Shink E, Bolam JP: Microcircuitry of the direct and indirect pathways of the basal ganglia. Neuroscience 1998; 86:353-387.

39 Stornetta RL, Rosin DL, Simmons JR, McQuiston TJ, Vujovic N, Weston MC, Guyenet PG: Coexpression of vesicular glutamate transporter-3 and gamma-aminobutyric acidergic markers in rat rostral medullary raphe and intermediolateral cell column. J Comp Neurol 2005;492:477-494.

40 Sze SCW, Wong CKC, Yung KKL: Modulation of the gene expression of N-methyl-Daspartate receptor NR2B in the rat neostriatum by a single dose of specific antisense oligodeoxynucleotide. Neurochem Int 2001; 39:319-327.

41 Takamori S, Malherbe P, Broger C, Jahn R: Molecular cloning and functional characterization of human vesicular glutamate transporter 3. EMBO Rep 2002;3:798-803.

42 Takamori S, Rhee JS, Rosenmund C, Jahn R: Identification of a vesicular glutamate transporter that defines a glutamatergic phenotype in neurons. Nature 2000;407:189-194.

43 Takamori S, Rhee JS, Rosenmund C, Jahn R: Identification of differentiation-associated brain-specific phosphate transporter as a second vesicular glutamate transporter (VGLUT2). J Neurosci 2001;21:RC182.

44 Ungerstedt U: 6-Hydroxy-dopamine induced degeneration of central monoamine neurons. Eur J Pharmacol 1968;5:107-110.

45 Ungerstedt U, Ljungberg T, Steg G: Behavioral, physiological, and neurochemical changes after 6-hydroxydopamine-induced degeneration of the nigro-striatal dopamine neurons. Adv Neurol 1974;5:421-426.

46 Varoqui H, Schafer MK, Zhu H, Weihe E, Erickson JD: Identification of the differentiation-associated $\mathrm{Na}^{+} / \mathrm{PI}$ transporter as a novel vesicular glutamate transporter expressed in a distinct set of glutamatergic synapses. J Neurosci 2002;22:142-155.

47 Wojcik SM, Rhee JS, Herzog E, Sigler A, Jahn R, Takamori S, Brose N, Rosenmund C: An essential role for vesicular glutamate transporter 1 (VGLUT1) in postnatal development and control of quantal size. Proc Natl Acad Sci USA 2004;101:7158-7163.

48 Yamada M, Oligino T, Mata M, Goss JR, Glorioso JC, Fink DJ: Herpes simplex virus vector-mediated expression of $\mathrm{Bcl}-2$ prevents 6 hydroxydopamine-induced degeneration of neurons in the substantia nigra in vivo. Proc Natl Acad Sci USA 1999;96:4078-4083. 